\title{
Aerodynamics of Stardust Sample Return Capsule
}

\author{
R. A. Mitcheltree*, R. G. Wilmoth† F. M. Cheatwood \\ G. J. Brauckmann, F. A. Greene, \\ NASA Langley Research Center, Hampton, Virginia
}

\begin{abstract}
Successful return of interstellar dust and cometary material by the Stardust Sample Return Capsule requires an accurate description of the Earth entry vehicle's aerodynamics. This description must span the hypersonic-rarefied, hypersonic-continuum, supersonic, transonic, and subsonic flow regimes. Data from numerous sources are compiled to accomplish this objective. These include Direct Simulation Monte Carlo analyses, thermochemical nonequilibrium computational fluid dynamics, transonic computational fluid dynamics, existing wind tunnel data, and new wind tunnel data. Four observations are highlighted: 1) a static instability is revealed in the free-molecular and early transitionalflow regime due to aft location of the vehicle's center-of-gravity, 2) the aerodynamics across the hypersonic regime are compared with the Newtonian flow approximation and a correlation between the accuracy of the Newtonian flow assumption and the sonic line position is noted, 3) the primary effect of shape change due to ablation is shown to be a reduction in drag, and 4) a subsonic dynamic instability is revealed which will necessitate either a change in the vehicle's center-of-gravity location or the use of a stabilizing drogue parachute.
\end{abstract}

\section{Introduction}

$\mathbf{S}^{\mathrm{T}}$ TARDUST ${ }^{1}$, the fourth Discovery class mission, is scheduled for launch in February of 1999. In addition to collecting interstellar dust, the robotic spacecraft will fly within $100 \mathrm{~km}$ of the comet Wild-2 nucleus and collect pre-solar cometary material from the coma parent-molecular zone. These materials will be returned to Earth for submicron level analysis. To accomplish the mission's objective, a capsule containing the collected particles must safely transit an intense Earth entry, descent, and landing. This paper focuses on the aerodynamics of the Stardust Sample Return Capsule (SRC) during that entry. The results also have relevance to other proposed sample return missions.

The entry of the Stardust SRC at $12.6 \mathrm{~km} / \mathrm{s}$ will be the fastest Earth entry ever attempted. Its trajectory traverses the hypersonic-rarefied, hypersonic-

*Aerospace Engineer, Aerothermodynamics Branch, Aeroand Gas-Dynamics Division, NASA Langley Research Center, Senior Member AIAA.

${ }^{\dagger}$ Aerospace Engineer, Aerothermodynamics Branch, Aeroand Gas-Dynamics Division, NASA Langley Research Center, Senior Member AIAA.

Aerospace Engineer, Vehicle Analysis Branch, Space Systems and Concepts Division, NASA Langley Research Center, Member AIAA.

$\S$ Aerospace Engineer, Aerothermodynamics Branch, Aeroand Gas-Dynamics Division, NASA Langley Research Center, Senior Member AIAA.

Aerospace Engineer, Aerothermodynamics Branch, Aeroand Gas-Dynamics Division, NASA Langley Research Center, Senior Member AIAA.

Copyright (C) 1997 by the American Institute of Aeronautics and Astronautics, Inc. No copyright is asserted in the United States under Title 17, U.S. Code. The U.S. Government has a royalty-free license to exercise all rights under the copyright claimed herein for governmental purposes. All other rights are reserved by the copyright owner. continuum, supersonic, transonic, and subsonic flow regimes. The passive capsule, once released from its host bus, will rely solely on the predetermined balance between aerodynamic forces and gravity to guide it through those regimes to a parachute landing, within a $75 \mathrm{~km}$ ellipse, in the Utah Test Landing Range. Highfidelity aerodynamic knowledge is essential for mission success. The drag coefficient must be accurately described within each flight regime so the cumulative effect of the deceleration results in a landing within the targeted Utah site. In addition, the capsule should possess sufficient aerodynamic stability to minimize angle-of-attack excursions during the severe heating portion of the trajectory. This stability must persist through the transonic and subsonic regimes to maintain a controlled attitude at parachute deployment.

The objective of this paper is to describe the aerodynamics of the Stardust SRC and assess if the requirements cited above are met. The description must be constructed with sufficient breadth and detail to populate an aerodynamic database suitable for six degree-of-freedom trajectory simulations. Data from numerous sources are compiled. These include Direct Simulation Monte Carlo (DSMC) analysis to describe the rarefied flow in the transitional regime, thermochemical nonequilibrium computational fluid dynamics (CFD) for the hypersonic regime, CFD and existing wind tunnel data in the supersonic and transonic regime, and finally, new subsonic static and dynamic wind tunnel test data.

A description of the entry capsule's geometry is presented first. Discussion of the approach taken to describe the static aerodynamics in each flight regime is next, followed by comments on the dynamics. Detailed description of the aerodynamics through each of the flight regimes requires knowledge of the expected 
trajectory. Three degree-of-freedom (3-DOF) simulations were computed to satisfy this need. From this estimated trajectory, discrete points are chosen for the high-fidelity analysis.

\section{Sample Return Capsule Geometry}

The forebody geometry of the SRC is a 60-degree half-angle sphere-cone with nose radius equal to 0.2286 $\mathrm{m}$, shoulder radius of $0.01905 \mathrm{~m}$, and overall diameter of $0.8128 \mathrm{~m}$. The afterbody shape is a 30-degree cone which terminates with a flat stern whose radius is $0.2116 \mathrm{~m}$. The geometry is shown in Figure 1. The forebody heat shield is made of PICA (Phenolic Impregnated Carbon Ablator). Surface recession at the nose due to heating rates as high as $1200 \mathrm{~W} / \mathrm{cm}^{2}$ is estimated to be $0.01194 \mathrm{~m}(0.47 \mathrm{in})$ and $0.006858 \mathrm{~m}(0.27$ in) at the shoulder. For aerodynamic considerations, this shape change due to ablation is assumed to result in a forebody which remains a 60-degree sphere cone except the nose radius increases to $0.2405 \mathrm{~m}$, shoulder radius increases to $0.02591 \mathrm{~m}$, and overall diameter decreases to $0.7991 \mathrm{~m}$. The ablative shape change impact on the aerodynamics is presented.

\section{Low-Density Aerodynamics}

The Stardust SRC is released from the host spacecraft 3 to 4 hours prior to atmospheric interface. When the spin-stabilized capsule arrives at the outer reaches of the atmosphere, it will encounter widely spaced molecules. Surface impacts of these molecules will exert the first aerodynamic forces on the entry vehicle. Knudsen number, $K n$, is defined as the ratio of the quiescent gas's mean free path to the vehicle's diameter. At the outer reaches of the atmosphere, $K n$ is large. As long as $K n>10$, the associated aerodynamic forces can be accurately computed by a free-molecular-flow method. Free-molecular flow assumes there are no collisions between gas molecules in the flow field. The surface is impacted by free-stream particles which are diffusely reflected after full thermal accommodation. Unlike hypersonic-continuum aerodynamics, where forces exerted on the blunt body are primarily the integrated effect of surface pressures, free-molecular-flow aerodynamics contain a significant contribution from shear stress.

Free-molecular-flow aerodynamics for the Stardust geometry are computed using a collisionless DSMC approach discussed in Ref. 2. The results are presented in Fig. 2. Since the geometry is axisymmetric, static aerodynamics can be described by the variation of axial force coefficient $\left(C_{A}\right)$, a normal-force coefficient $\left(C_{N}\right)$, and a moment coefficient $\left(C_{m}\right)$ with respect to angle-of-attack $(\alpha)$. The reference area is the frontal area of the vehicle $\left(0.51887 \mathrm{~m}^{2}\right)$, and the reference length is the diameter $(0.8128 \mathrm{~m})$. Unless otherwise specified, moments are taken about the nose of the vehicle.
Table 1 Axisymmetric Results from DSMC (Ref. 3 method).

\begin{tabular}{||r||r|c|c||c||}
\hline $\mathrm{t}(\mathrm{s})$ & $\mathrm{Alt}(\mathrm{km})$ & $\mathrm{M}$ & $\mathrm{V}(\mathrm{m} / \mathrm{s})$ & $C_{A}$ \\
\hline 0 & 134.7 & - & 12597 & 2.019 \\
8 & 120.5 & - & 12608 & 1.974 \\
20 & 100.9 & - & 12620 & 1.822 \\
26 & 92.0 & 44.1 & 12618 & 1.640 \\
32 & 83.7 & 42.7 & 12592 & 1.543 \\
38 & 76.0 & 42.2 & 12487 & 1.525 \\
\hline
\end{tabular}

Table 2 3-D Results from DSMC (Ref. 4 method).

\begin{tabular}{||c||r||r||c|c|c||}
\hline $\mathrm{t}(\mathrm{s})$ & Alt $(\mathrm{km})$ & $\alpha$ & $C_{A}$ & $C_{N}$ & $C_{m}$ \\
\hline 20 & 100.9 & 0 & 1.86303 & 0. & 0. \\
20 & 100.9 & 10 & 1.80815 & 0.219899 & -0.07736 \\
\hline 32 & 83.7 & 0 & 1.60761 & 0. & 0. \\
32 & 83.7 & 10 & 1.52884 & 0.138041 & -0.03952 \\
\hline
\end{tabular}

As the capsule continues its descent to altitudes at or below $130 \mathrm{~km}, K n$ drops below 10 and collisions between molecules become important and must be included in aerodynamic predictions. This is the transitional flow regime.

For a given $K n$, the transitional-regime aerodynamics can be described by Direct Simulation Monte Carlo (DSMC) methods ${ }^{2-6}$. Three separate efforts are used to compute the transitional-regime aerodynamics for the Stardust SRC. First, six zero-degree angle-ofattack cases are computed using the method of Ref. 3 . The predictions of $C_{A}$ are presented in Table 1 . The three-dimensional DSMC methods of Rault et. al. ${ }^{4}$ and Wilmoth et. al. ${ }^{5}$ are applied at points within the transitional portion of the trajectory. The results are presented in Table 2 and Table 3 . In general, there is good agreement between the different DSMC methods. The scatter in the results for moment coefficient at 83 $\mathrm{km}$ is indicative of the uncertainty DSMC (and CFD) suffer at this $K n$ and is also the result of differences in computational approaches and grids.

Predictions from the three DSMC solutions at zero degrees angle-of-attack for axial coefficient are shown in Fig. 3. The plot also contains the free-molecular value, and three hypersonic-continuum predictions (from CFD calculations discussed subsequently).

A bridging function selected as appropriate to recreate the data within the $k n$ range 10.0 to 0.001 is included in Fig. 3. The bridging function formula for the axial coefficient is;

$$
C_{A}=C_{A, c}+\left(C_{A, f m}-C_{A, c}\right) \sin ^{2}(\phi)
$$


Table 3 3-D Results from DSMC (Ref. 5 method).

\begin{tabular}{||c||r||r||c|c|c||}
\hline $\mathrm{t}(\mathrm{s})$ & $\mathrm{Alt}(\mathrm{km})$ & $\alpha$ & $C_{A}$ & \multicolumn{1}{c|}{$C_{N}$} & $C_{m}$ \\
\hline 0 & 134.7 & 0 & 2.039 & 0. & 0. \\
0 & 134.7 & 10 & 1.961 & 0.3294 & -0.0875 \\
0 & 134.7 & 30 & 1.541 & 0.8432 & -0.2234 \\
\hline 20 & 100.9 & 0 & 1.831 & 0. & 0. \\
20 & 100.9 & 10 & 1.772 & 0.2330 & -0.0738 \\
20 & 100.9 & 30 & 1.403 & 0.5896 & -0.2050 \\
\hline 32 & 83.7 & 0 & 1.553 & 0. & 0. \\
32 & 83.7 & 10 & 1.479 & 0.1244 & -0.0505 \\
32 & 83.7 & 30 & 1.097 & 0.3299 & -0.1671 \\
\hline
\end{tabular}

where

$$
\phi=\pi\left(\frac{3}{8}+\frac{1}{8} \log _{10} k n\right)
$$

$C_{A, f m}$ and $C_{A, c}$ are the values of the axial coefficient at the free molecular and continuum limits. This equation approximates the monotonic decrease in $C_{A}$ across the transitional regime. Ideally, bridging functions could be used to describe the variation of $C_{A}$, $C_{N}$, and $C_{m}$ across a large angle-of-attack range in this regime. This would eliminate the need to compute a large matrix of DSMC solutions. Figures 45 present the normal-force and moment coefficients at 10 degree angle-of-attack compared with bridging functions analogous to Eqs. 1-2. Note, the bridging functions yield good agreement with the $C_{A}\left(\alpha=0^{0}\right)$ and $C_{N}\left(\alpha=10^{\circ}\right)$ results, but are ill-suited for the non-monotonicity of the moment coefficient. (If the moment coefficient were taken about the center-ofgravity location rather than the nose, the variation of $C_{m}$ with $K n$ is monotonic.)

Figure 6 shows the shift in the center-of-pressure (c.p.) location across the transitional regime. The c.p. is at 0.26 body diameters $(0.26 \mathrm{D})$ back from the nose in the free-molecular limit. This location is well forward of the continuum value which is $0.72 \mathrm{D}$. For the vehicle to be statically stable, the center-of-gravity (c.g.) must be located ahead of the c.p. Stardust (with its c.g. currently specified at $0.35 \mathrm{D}$ ) is unstable in the free-molecular-flow region. Figure 7 confirms this fact by presenting the expected moment coefficient about that c.g. location at different Knudsen numbers. The figure reveals that at the highest altitudes (freemolecular limit), the capsule will seek to trim at 60 degrees incidence. It becomes stable about $\alpha=0^{0}$ when $K n$ drops below 0.09 .

The decrease in stability with increasing rarefaction (i.e., increasing $K n$ ) is a result of the increased shear stress contribution to the aerodynamic forces. This trend has been observed in shuttle flights and documented for the blunt Soyuz capsule by Ivanov ${ }^{6}$. The divergence between continuum stability and freemolecular stability increases with bluntness (i.e., cone half-angle) as shown in Fig. 8.

The question arises as to whether the gyroscopic stability of the spinning capsule (originally at five rotations-per-minute) will be sufficient to retard the destabilizing aerodynamic forces until the capsule has passed through the altitudes at which the capsule is unstable.

The aerodynamic forces are proportional to the dynamic pressure. In the free-molecular-flow regime, dynamic pressures are usually small because of low atmospheric densities. Stardust SRC, however, is smaller than past entry vehicles. Free-molecular and transitional flow conditions, therefore, persist to lower altitudes than previous vehicles experienced. Lower altitudes correspond to higher densities. In addition, the entry velocity at $12.6 \mathrm{~km} / \mathrm{s}$ is higher than any other Earth entry. These two factors combine to result in non-negligible dynamic pressures (and thus aerodynamic forces) in the rarefied flow regimes. Furthermore, the interior of the capsule is somewhat empty so its rotational inertia (and thus gyroscopic stability even at five rotations-per-minute) is small. Preliminary six-degree-of-freedom (6-DOF) calculations indicate that the gyroscopic stability is not sufficient to retard the destabilizing aerodynamic forces. As a result, the capsule's angle-of-attack will increase from its desired zero degree orientation towards the 60-degree trim point early in the trajectory. Large angles of incidence are worrisome since the capsule is stable flying backwards. Off-nominal attitudes or pitch rates at atmospheric interface could result in a backwards entry. In addition, large incidence angles early in the flight can result in incidence angles above 10 degrees at peak heating. Large angles-of-attack dramatically increase the afterbody heating near the shoulder regions and may damage the afterbody thermal protection system. Further discussion of the low-density aerodynamics of the $\mathrm{SRC}$ are included in Wilmoth et. al. ${ }^{2}$.

\section{Hypersonic Aerodynamics}

As the capsule continues its descent below $66 \mathrm{~km}$ attitude, $K n$ drops below 0.001 and continuum methods can be used to describe the flow about (and the forces on) the capsule. In this hypersonic portion of the entry, the flowfield is dominated by a strong bow shock. Forebody pressures are two orders of magnitude larger than afterbody pressures. The afterbody can, therefore, be neglected when computing the aerodynamic characteristics at small angles-of-attack in this regime. The Langley Aerothermodynamics Upwind Relaxation Algorithm (LAURA) CFD code ${ }^{7}$ is used to compute solutions at nine points in the trajectory's hypersonic regime. LAURA is an upwind-biased, point-implicit relaxation algorithm for obtaining the numerical solution to the Navier-Stokes equations for three dimensional, viscous, hypersonic flows in thermochemical nonequilibrium ${ }^{8}$. It has been used to describe the 
Table 4 Axisymmetric Results from LAURA.

\begin{tabular}{||l||l|c|c||l||}
\hline $\mathrm{t}(\mathrm{s})$ & $\mathrm{Alt}(\mathrm{km})$ & $\mathrm{M}$ & $\mathrm{V}(\mathrm{m} / \mathrm{s})$ & $C_{A}$ \\
\hline 32 & 83.7 & 42.7 & 12592 & 1.5636 \\
44 & 68.96 & 40. & 12173 & 1.4959 \\
53.2 & 58.7 & 35.4 & 10983 & 1.4828 \\
64 & 50.63 & 24.7 & 7902 & 1.4816 \\
72 & 46.54 & 17.1 & 5496 & 1.4939 \\
78 & 45.75 & 12.2 & 3984 & 1.4992 \\
82 & 44.44 & 10.5 & 3289.0 & 1.515 \\
86 & 43.24 & 8.5 & 2724.5 & 1.510 \\
92 & 41.60 & 7.15 & 2076.2 & 1.506 \\
\hline
\end{tabular}

Table 5 3-D Results from LAURA.

\begin{tabular}{|c||c||c||c||c||c|}
\hline $\mathrm{t}(\mathrm{s})$ & $\mathrm{M}$ & $\alpha$ & $C_{A}$ & $C_{N}$ & $C_{m}$ \\
\hline 32. & 42.7 & 10. & 1.4807 & 0.10903 & -0.05483 \\
\hline 44. & 40.5 & 10. & 1.4455 & 0.08874 & -0.06308 \\
\hline 53.2 & 35.4 & 5. & 1.4740 & 0.04476 & -0.03206 \\
53.2 & 35.4 & 10. & 1.4338 & 0.08650 & -0.06136 \\
\hline 64.0 & 24.7 & 5. & 1.4708 & 0.04342 & -0.03176 \\
64.0 & 24.7 & 10. & 1.4303 & 0.08330 & -0.05996 \\
\hline 72 & 17.1 & 5. & 1.4806 & 0.04451 & -0.03224 \\
72 & 17.1 & 10. & 1.4208 & 0.07461 & -0.05223 \\
\hline 78 & 12.2 & 5. & 1.4889 & 0.04452 & -0.03220 \\
78 & 12.2 & 10. & 1.4242 & 0.07508 & -0.05172 \\
\hline 82 & 10.5 & 5. & 1.498 & 0.04074 & -0.02822 \\
\hline 86 & 8.5 & 5. & 1.496 & 0.03036 & -0.01895 \\
\hline 92 & 7.15 & 5. & 1.477 & 0.02270 & -0.01309 \\
\hline
\end{tabular}

aerodynamics of several blunt bodies including Mars Pathfinder ${ }^{9}$. Table 4 presents the zero-degree angleof-attack $C_{A}$ results. Three-dimensional solutions at 5 and 10 degrees angle-of-attack are computed for the forebody at the nine trajectory points (Table 5 ).

For continuum conditions with Mach numbers above 12,11 species thermochemical nonequilibrium effects are included. At and below Mach 12, the flow is assumed to be in chemical equilibrium. The axisymmetric solutions are computed on shock-aligned grids with 30 points along the forebody and 64 points normal to the surface. The cell Reynolds number for the first cell off the wall is unity. Three-dimensional solutions utilize an axis-singularity-free grid with a 58 by 26 cell surface definition. Confidence in these computational grids for accurately resolving surface pressures stems from previous experiences ${ }^{10,11}$ on similar geometries.

Figure 9 presents the LAURA calculations for zero angle-of-attack axial coefficient. Little variation in the predicted values occurs across the entire hypersoniccontinuum regime (above Mach 38 is the transitional regime where $C_{A}$ increases as was shown in Fig 3). A Newtonian flow prediction for the capsule is in- cluded as the dashed line. (Newtonian flow assumes that the free-stream flow is turned parallel to the surface. Local pressure is then a function only of the local surface's inclination angle to the free stream.) Figure 10 presents the variation of the axial coefficient with angle-of-attack for CFD predictions above Mach 12 . Increased angle-of-attack results in a small decrease in axial force across the Mach range.

Figures 11 and 12 display predicted normal-force and moment coefficients as a function of Mach number from 35 to 7 at 5 and 10 degrees angle-of-attack. The marked decrease in normal-force and increase in moment coefficients below Mach 12 is a result of the sonic line shifting from the sphere-cone tangency point to a location on the shoulder of the 60-degree forebody cone. This shift occurs as the flowfields exhibit less real-gas effects and begin to resemble ideal-gas flows. Pressure distributions (and thus aerodynamic forces) are affected by the sonic character of the shock layer. When supersonic, the pressure distributions on the conical flank are flat, which is characteristic of conical flow. When the entire forebody shock layer is subsonic, the elliptic nature of that flow results in higher, more rounded distributions. Figures 10-12 show that the Newtonian flow approximation is accurate only when the sonic line resides on the spherical nose. The net effect of the sonic line shift below Mach 12 is a decrease in the static stability margin (the c.p. moves forward to $0.58 \mathrm{D}$ back from the nose).

The hypersonic regime aerodynamics are complicated by forebody shape change due to ablation. Thus far, the discussion of hypersonic aerodynamics has excluded this shape change. As the vehicle encounters heating as high as $1200 \mathrm{~W} / \mathrm{cm}^{2}$, the PICA forebody heatshield ablates and begins to recede. Figure 13 presents the predicted recession at the nose and the shoulder for the Stardust overshoot trajectory. Recession begins in the transitional-flow regime and continues down to Mach 7.6. Surface recession decreases the frontal area (i.e., diameter) and increases the nose radius. Figure 13 supplied the information used to describe the ablated shape discussed in the Sample Return Capsule Geometry section. A LAURA solution is generated on the ablated shape (Mach 35.4). A comparison of forebody pressures and axial coefficients for the original and ablated geometry as predicted by LAURA is presented in Fig. 14. The shape change results in a decrease in axial coefficient of 0.8 percent. This change is primarily a result of the ablated shape's rounder shoulder. A Newtonian flow approximation of the ablated shape predicts a 0.6 percent decrease in the axial coefficient.

A comparison of the normal-force and moment coefficients between the nonablated and ablated shape shows little change. These aerodynamic coefficients are, however, referenced to their respective areas, diameters, and nose locations. The total drag on the 
vehicle will decrease with ablation shape change more than the 0.8 percent indicated as a result of the decrease in reference area. In creating an aerodynamic data base, these reference shifts must be tracked accurately.

\section{Supersonic Aerodynamics}

Below Mach 7 , forebody-only CFD will not accurately predict the aerodynamics. The calculations must include the afterbody and wake. Such calculations are computationally expensive. Fortunately, wind tunnel data for configurations similar to the SRC exist. In the low hypersonic and supersonic region, Walker ${ }^{12}$ measured the static aerodynamics on blunted 60-degree cones in the JPL 20-in. supersonic wind tunnel. The Reynolds number for these tests, based on diameter, is 4.0 million. (The expected flight Reynolds numbers in this speed regime are around 0.2 million.) The 60-degree sphere-cone models Walker tested possessed a range of shoulder radii which envelopes the expected Stardust value. He performed measurements on a sharp-shouldered model and one with a shoulder radius equal to 5 percent of the base radius. The Stardust ablated geometry has a shoulder radius at 3.25 percent of its base radius. Unfortunately, the nose bluntness of his models ( 30 percent of the base radius) is not as large as the Stardust ablated geometry's (59 percent of the base radius) and the wind tunnel models had no afterbody.

Figures $15-17$ present $C_{A}, C_{N}$, and $C_{m}$ as a function of Mach number for $\alpha=5^{0}$. (The transonic and subsonic data in the plots are discussed later.) In the supersonic regime, the figures indicate that little change in the aerodynamic coefficients occurs between Mach 7.15, where the last LAURA solution is generated, and Mach 4 where the Walker data begins. While Mach 3.98 is the highest value Walker tests for the models closest to Stardust, he did examine similar 60-degree cones up to Mach 9.5 in the JPL 21-in hypersonic wind tunnel. His results show no change in the aerodynamics between Mach 9.5 and Mach 4 . He also compared his Mach 9.5 results to modified Newtonian predictions and saw poor agreement. This observation agrees with earlier results displayed in Figs. 9-12 which revealed the Newtonian approximation to be accurate only down to Mach 12 where the sonic-line shift occurs. Note, the solid line in Figs 15-17 labeled "database" is included to indicate trends.

\section{Transonic Aerodynamics}

Wind tunnel measurements examining the same 60degree wind tunnel models Walker examined at supersonic speeds were performed at transonic conditions by Marko in the NASA Ames 2 by 2 Transonic wind tunnel ${ }^{13}$. The data were measured at a Reynolds number based on diameter of 1.0 million; the flight value is near 0.2 million. These measurements are included in Figs. 15-17. Marko did not include the moment coefficient measurements in his report. He included c.p. locations at a subset of his test conditions which, combined with the normal coefficient data, were used to compute the moment coefficients shown in Fig. 17. Wind tunnel testing around Mach 1.0 is difficult due to reflected shock interference. Though Marko does not suggest this as a potential source of errors in his measurements, the data contain oscillations in $C_{N}$ and $C_{m}$ at Mach 1.0 .

In an attempt to augment the definition of the transonic region aerodynamics, the CFD code TLNS3D (Thin Layer Navier Stokes 3-Dimensional) ${ }^{14}$ was used to examine the flight conditions between Mach 0.6 and 2.0. The results are included in Figs 15-17. Some questions exist as to the accuracy of these CFD solutions. The concerns center around computation of the wake flow. The TLNS3D solver requires a turbulence model to be used in order to avoid numerical instabilities in the massive recirculation wake zone. However, none of the available turbulence models are accurate in such a separated flow region. Extensive grid resolution studies were performed, but questions remain as to the solution's accuracy. The largest question in the CFD predictions occurs in $C_{N}$ though not shown, the predicted value at $\mathrm{M}=0.6$, and $\alpha=5$ degrees is negative.

\section{Subsonic Aerodynamics}

The Stardust SRC entry scenario planned to deploy a parachute at $3 \mathrm{~km}$ altitude corresponding to a Mach number of 0.16 . The deployment occurs 400 seconds after atmospheric interface. Of these $400 \mathrm{sec}-$ onds of entry, 200 are spent at Mach numbers less than 0.6 . The authors were not aware of any existing subsonic wind tunnel data on the SRC shape. A wind tunnel investigation of the Stardust SRC $(0.30$ scale ablated shape geometry) was conducted at Mach 0.16. The force-and-moment model of the SRC was constructed from high density foam and covered with a fiberglass skin. Hard points within the structure were reinforced with aluminum and wood. The model was sting mounted to a six component balance through the base. The after-body conical portion was fitted with 6 flush mounted pressure orifices in a longitudinal ray. (These pressure taps were incorporated to supply information necessary to calibrate the barometric parachute-deploy switch.)

The model was tested in the ViGYAN Low Speed Wind Tunnel in Hampton, Virginia. This tunnel is a conventional, straight-through, open-return type layout with a 3' by 4' open-jet test section. The model was attached to an angle-of-attack mechanism which was swept from 0 to 28 degrees inclination. Balance data were reduced to coefficient form accounting for balance interactions, sting deflections, and model cavity pressures. The tunnel was run at a dynamic 
Table 6 Vigyan Subsonic Data $(M=0.16)$.

\begin{tabular}{|c|c|c|c|}
\hline$\alpha$ & $C_{A}$ & $C_{N}$ & $C_{m}$ \\
\hline 0.00 & 0.8739 & 0.0000 & 0.0000 \\
2.02 & 0.8759 & 0.0053 & -0.0058 \\
4.00 & 0.8779 & 0.0119 & -0.0115 \\
6.04 & 0.8794 & 0.0177 & -0.0171 \\
7.99 & 0.8809 & 0.0242 & -0.0227 \\
12.02 & 0.8838 & 0.0357 & -0.0332 \\
16.01 & 0.8822 & 0.0485 & -0.0446 \\
20.00 & 0.8774 & 0.0613 & -0.0573 \\
24.01 & 0.8607 & 0.0723 & -0.0676 \\
28.01 & 0.8212 & 0.0855 & -0.0757 \\
\hline
\end{tabular}

pressure of $0.018 \mathrm{~atm}$. (The expected flight dynamic pressure is 0.012 atm.) Reynolds numbers based on diameter for the tests were 0.9 million. The resulting aerodynamic coefficients are listed in Table 6 and plotted in figures $18-20$ as a function of angle-of-attack. Note, the normal-force and moment coefficients may be considered linear over the angle-of-attack range examined. The data from these tests are also included at the far left hand side of Figs. 15-17. The laminar or turbulent nature of the boundary layer for the flight case is not known, nor is it known if the wind tunnel test case was turbulent.

\section{Dynamic Derivatives}

Accurate six degree-of-freedom trajectory simulations of the SRC entry require knowledge of the dynamic stability of the capsule. This requirement is especially true in the transonic and subsonic speed regimes.

Uselton et al. ${ }^{15}$ examined the dynamic stability of blunted 60-degree and 70-degree cones at Mach numbers between 3.0 and 0.6 . He demonstrated that such shapes can suffer a dynamic instability at small angles-of-attack in the transonic flight regime. That is, though they remain statically stable, when Mach number decreases below 2.0 an increase in incidence angles (a wobbling motion) may occur. Bendura ${ }^{16}$ examined the dynamic stability of blunted 60-degree cones in the low subsonic regime and revealed a sensitive dependence on c.g. location. The desire for Stardust is that the capsule remain in a controlled flight through these speed regimes and that large oscillations do not exist at the Mach 0.16 parachute deployment.

Since the capsule spends the last 200 seconds of its entry at subsonic conditions, its attitude at parachute deploy is influenced most by its subsonic dynamic stability. To establish this property of the capsule, low subsonic dynamic stability tests were conducted in the 20 Foot Spin Tunnel at Langley Research Center. The tests revealed that the configuration was dynamically unstable at the conditions of the test due to the aft-location of the c.g. ( $0.35 \mathrm{D}$ back from the nose).
Oscillations grew rapidly and diverged until the capsule began tumbling. If the c.g. was moved forward to $0.29 \mathrm{D}$, the divergent behavior was eliminated and the capsule established itself in a limit cycle oscillation with amplitude near 10 degrees.

The addition of different sized drogue parachutes was examined as a means of stabilizing the original c.g. location configuration. A parachute with drag area of at least $0.208 \mathrm{~m}^{2}$ was required to damp large perturbations. Details of these tests are discussed in Ref. 17.

At hypersonic speeds, ballistic range tests ${ }^{18}$ do not discern significant changes in the values of the dynamic derivatives with Mach number. In addition, past experience with 6-DOF simulations have also indicated that large variations in the dynamic derivatives at the higher Mach numbers have little effect on the hypersonic-flight dynamics of the vehicle.

\section{Conclusions}

Successful return of interstellar dust and cometary material by the Stardust Sample Return Capsule requires an accurate description of the Earth entry vehicle's aerodynamics. This description must span the hypersonic-rarefied, hypersonic-continuum, supersonic, transonic, and subsonic flow regimes. Data from numerous sources are compiled. These include Direct Simulation Monte Carlo analyses, thermochemical nonequilibrium CFD, transonic CFD, existing wind tunnel data, and new wind tunnel data.

A static instability is revealed in the free-molecular and early transitional-flow regime. The high entry velocity, small size, and low rotational inertia of the capsule combine to allow this instability to introduce large angles-of-attack during the high-altitude portion of the entry. In the extreme, this instability could result in a rear-facing entry of the capsule. Alleviation of the instability requires either 1) an alteration to the capsule's geometry, 2) a substantial increase in the spin rate from its original five rotations-per-minute specification, or 3 ) a repositioning of the center-of-gravity from its current location at 0.35 diameters(D) back from the nose to a position closer to $0.26 \mathrm{D}$.

In the transitional-flow regime, a simple monotonic bridging function (spanning the $K n$ range from 0.001 to 10.0 ) can be used to describe the variation of the axial and normal-force coefficients from their freemolecular value to their continuum value. This same approach does not appear accurate for the moment coefficient's non-monotonic variation (when moments are taken about the nose).

In the hypersonic-continuum regime, CFD solutions reveal that the Newtonian flow assumption is reasonably accurate as long as the sonic line remains on the vehicle's spherical nose. This is true for angles-ofattack of 5 degrees or less down to a Mach number of 12 . 
The sonic line shift from the spherical nose to the shoulder region below Mach 12 is accompanied by a lorward movement of the center-of-pressure $10.72 \mathrm{D}$ to $0.58 \mathrm{D})$ which decreases the static stability of the vehicle. This decrease in static stability is smaller than the one associated with the transition from continuum to free-molecular flow $(0.72 \mathrm{D}$ to $0.26 \mathrm{D})$.

The primary effect of ablation shape change on the vehicle's aerodynamics is a decrease in the drag coefficient resulting from the rounding of the shoulders and decreased frontal area. Uncertainties in the degree of shape change will translate directly into uncertainties in the landing foot print.

Finally, a dynamic instability in the subsonic regime will result in a tumbling motion in the terminal portion of the trajectory. To facilitate a successful parachute deployment at $\mathrm{M}=0.16$ and $3 \mathrm{~km}$ altitude, the centerof-gravity should be moved forward to 0.29 body diameters back from the nose or a stabilizing drogue chute, with drag area at least $0.208 \mathrm{~m}^{2}$, should be deployed prior to onset of subsonic speeds.

\section{Acknowledgments}

Dr. Y. K. Chen of NASA Ames Research Center supplied the surface recession estimate. Mr. William Willcockson of Lockheed-Martin Astronautics supplied the 3-DOF estimated trajectory. Prasun Desai of NASA LARC was helpful in many discussions and performed 6-DOF simulations which revealed the static instability in the transitional-flow regime. Thanks are also extended to Drs. J. N. Moss and D. F. Rault (LARC) for supplying additional DSMC solutions and W. A. Wood (LARC) for several LAURA CFD solutions.

\section{References}

${ }^{1}$ Atkins, K. L., Brownlee, D. E., Duxbury, T., Yen, C., and Tsou, P., "STARDUST: Discovery's Interstellar Dust and Cometary Sample Return Mission," Proceedings from the 1997 IEEE Aerospace Conference, Feb., 1997.

${ }^{2}$ Wilmoth, R. G., Mitcheltree, R. A., "Low-Density Aerodynamics of the Stardust Sample Return Capsule," AIAA Paper 97-2510, June, 1997.

${ }^{3}$ Bird, G. A., "The G2/A3 Program Users Manual," G.A.B. Consulting Pty Ltd., Killara, N.S.W., Australia, March,1992.

${ }^{4}$ Rault, D.F., Cestero, F.J, Shane, R.W., "Spacecraft Aerodynamic Characteristics During Aerobraking Maneuver in Planetary Atmospheres," AIAA Paper 96-1890.

${ }^{5}$ Wilmoth, R.G., LeBeau, G. J., and Carlson, A. B., "DSMC Grid Methodologies for Computing LowDensity, Hypersonic Flows About Reusable Launch Vehicles," AIAA Paper 96-1812, June 1996.

'Ivanov, M. S., Markelov, G.N., Gimelshein, S. F., and Antonov, S. G., "DSMC Studies of High-
Altitude Aerodynamics of Reentry capsule," 20th International Symposium on Rarefied Gas Dynamics, Beijing, China, Aug, 1996.

${ }^{7}$ Cheatwood, F. M., Gnoffo, P. A., "User's Manual for the Langley Aerothermodynamic Upwind Relaxation Algorithm (LAURA)," NASA TM 4674, Apr., 1996.

${ }^{8}$ Gnoffo, P. A., Gupta, R. N., and Shinn, J. L., "Conservation Equations and Physical Models for Hypersonic Air Flows in Thermal and Chemical Nonequilibrium," NASA TP-2867, Feb. 1989.

${ }^{9}$ Braun, R. D., Powell, R. W., Engelund, W, C., Gnoffo, P. A., Weilmuenster, K. J., and Mitcheltree, R. A., "Mars Pathfinder Six-Degree-of-Freedom Entry Analysis," J. of Spacecraft and Rockets, Vol 32, No. 6, Nov.-Dec., 1995, pp. 993-1000.

${ }^{10}$ Gnoffo, P. A., "Computation of Near-Wake, Aerobrake Flowfields," J. of Spacecraft and Rockets, Vol. 29, No. 2, Mar-Apr, 1992. pp. 182-189.

${ }^{11}$ Nettelhorst, H. L., Mitcheltree, R. A., "Grid Resolution and Solution Convergence for Mars Pathfinder Forebody," NASA TM 1.09173, Dec., 1994.

${ }^{12}$ Walker, B. and Weaver, R. W., "Static Aerodynamic Characteristics of Blunted Cones in the MachNumber Range from 2.2 to 9.5 ", JPL TR-32-1213, Dec. 1967.

${ }^{13}$ Marko, W. J., "Static Aerodynamic Characteristics of Three Blunted Sixty Degree Half-Angle Cones at Mach Numbers from 0.6 to $1.3, "$ TR 32-1298, JPL, July, 1968

${ }^{14}$ Vatsa, V. N., Turkel, E., and Abolhassani, J. S., "Extension of Multigrid Methodology to Supersonic/Hypersonic 3-D Viscous Flows," NASA Contractor Report 187612, Aug., 1991.

${ }^{15}$ Uselton B., L., Shadow, T. O., Mansfield, A. C., "Damping in Pitch Derivatives of 120 and 140 Deg Blunted Cones at Mach Numbers 0.6 through 3.0," AEDC TR-70-49. 1970.

${ }^{16}$ Bendura, R. J., "Low Subsonic Static and Dynamic Stability Characteristics of Two Blunt $120^{\circ}$ Cone Configurations," NASA TN D-3853, Feb, 1967.

${ }^{17}$ Mitcheltree, R. A., Fremaux, C. M., "Subsonic Dynamics of Stardust Sample Return Capsule," NASA TM 110329, March 1997.

${ }^{18}$ Krumins, Maigonis V., "A Ballistic Range Study of the Aerodynamic Characteristics of Mars Probe/Lander Shapes," AIAA Paper 67-167, Jan 1967. 


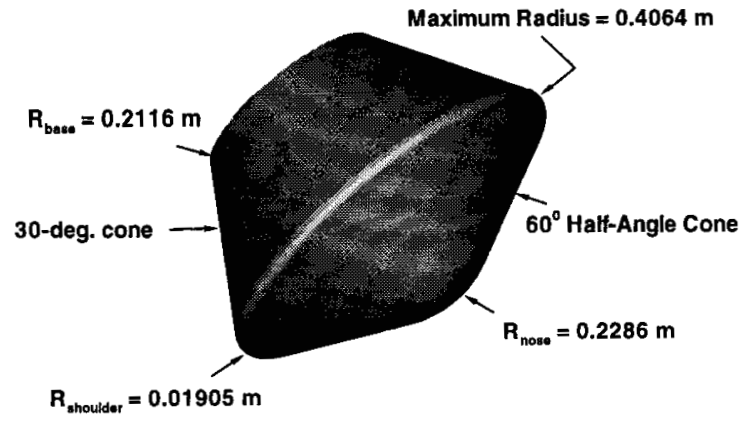

Fig. 1 Stardust Sample Return Capsule geometry.

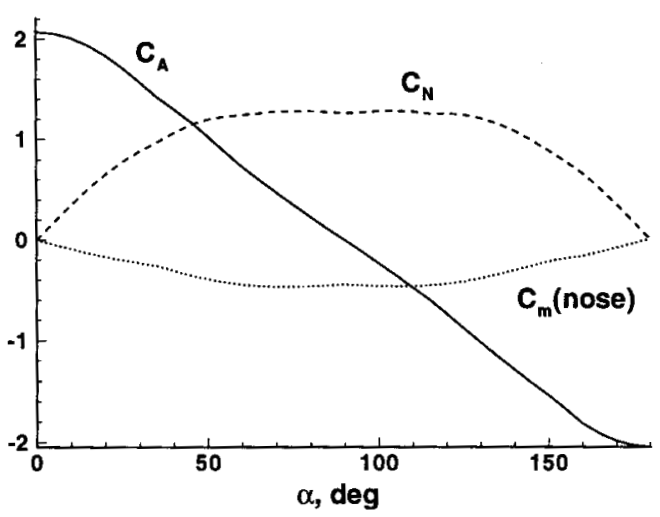

Fig. 2 Free-molecular-flow aerodynamics.

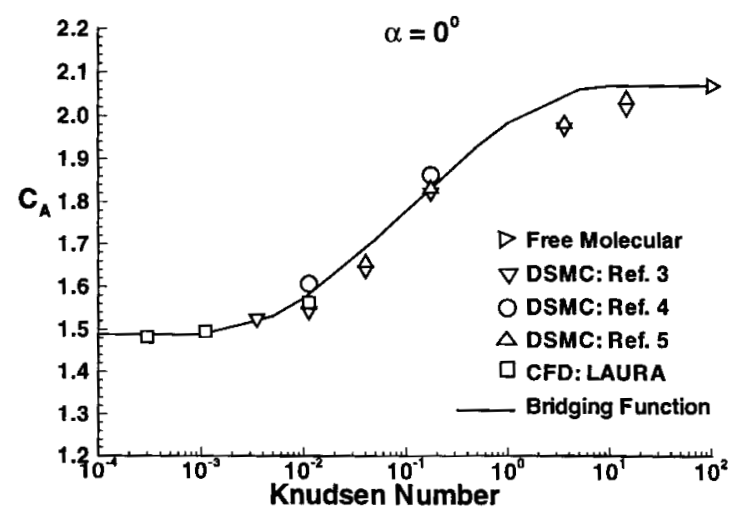

Fig. 3 Variation of the axial-force coefficient in the transitional-flow regime.

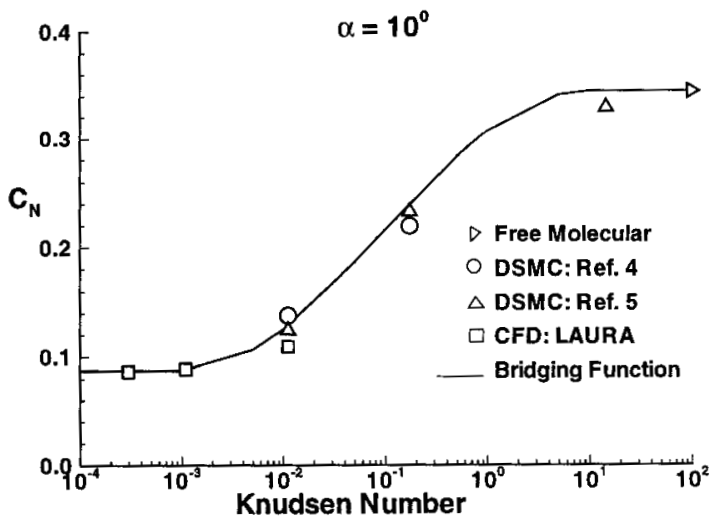

Fig. 4 Variation of the normal-force coefficient in the transitional-flow regime.

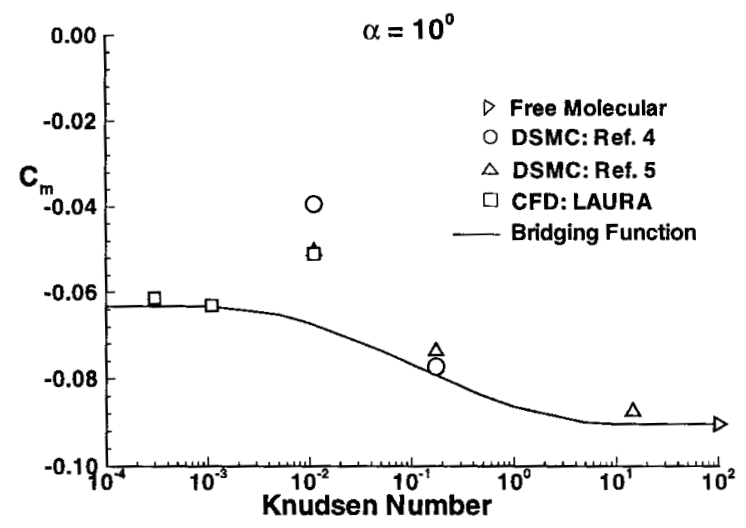

Fig. 5 Variation of the moment coefficient in the transitional-flow regime.

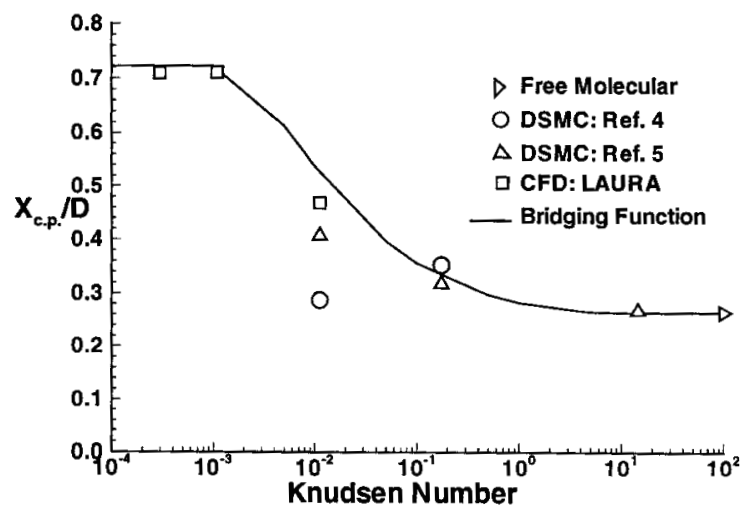

Fig. 6 Variation of the center-of-pressure location in the transitional-flow regime. 


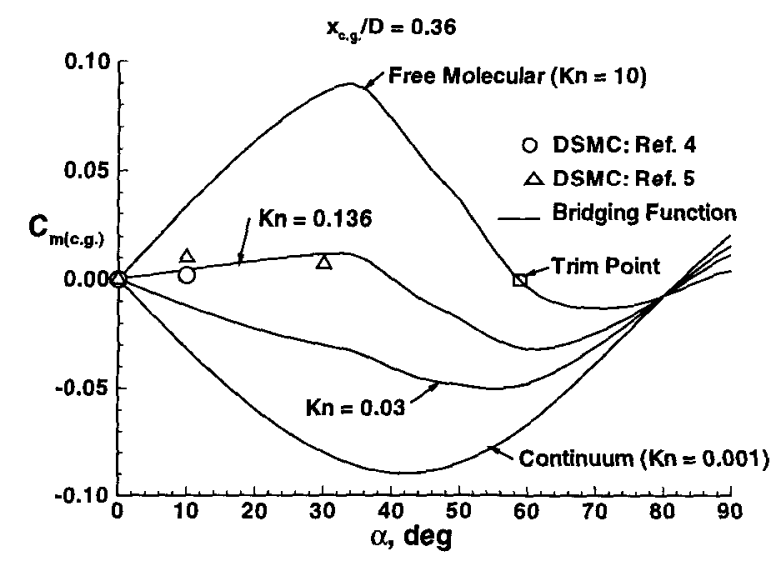

Fig. 7 Moment coefficient variation with angle-ofattack at different Knudsen numbers.

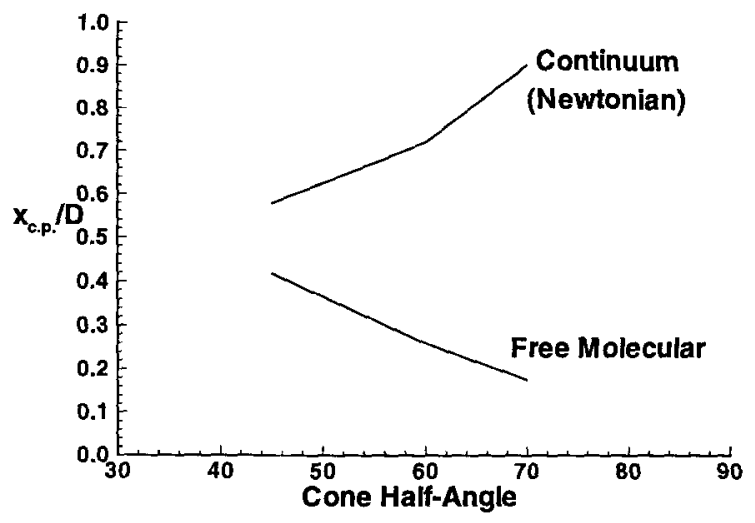

Fig. 8 Comparison of center-of-pressure location for various cone half-angles.

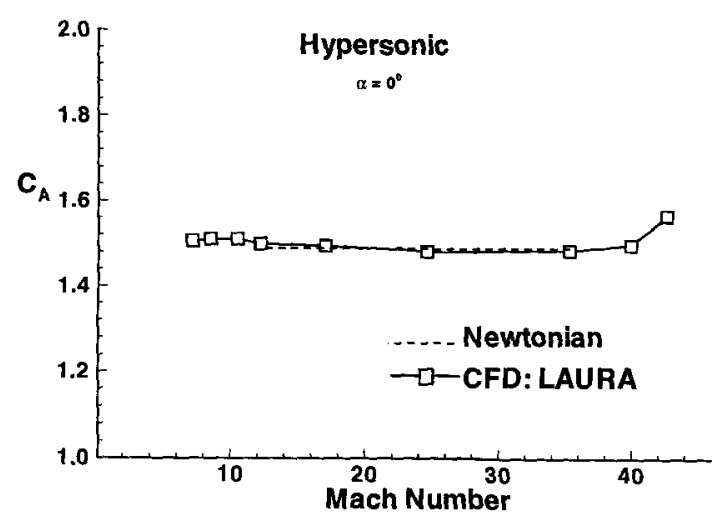

Fig. 9 Variation of axial-force coefficients in the hypersonic-flow regime.

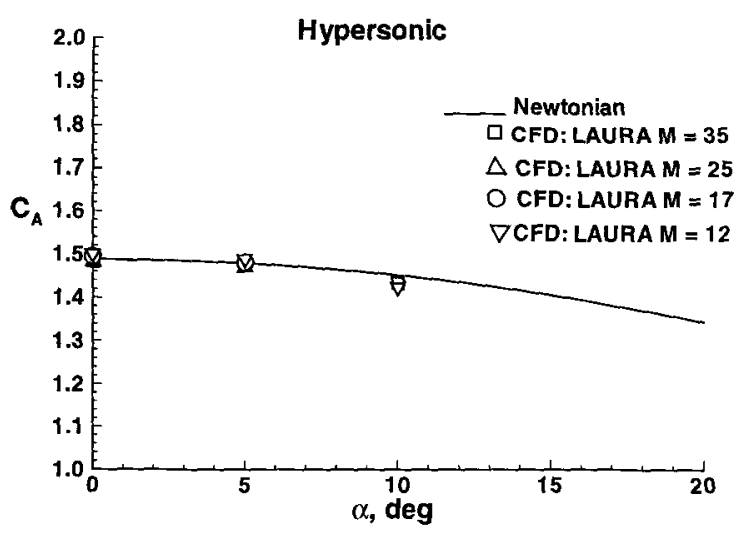

Fig. 10 Variation of axial-force coefficient with angle-of-attack in the hypersonic-flow regime.

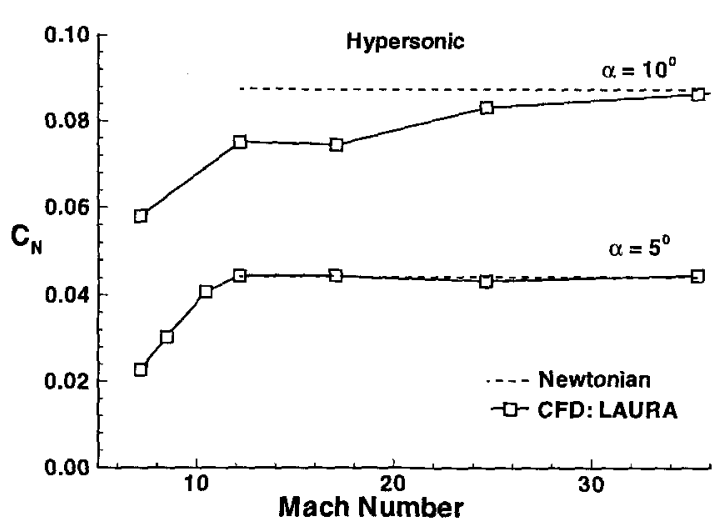

Fig. 11 Variation of normal-force coefficients in the hypersonic-flow regime.

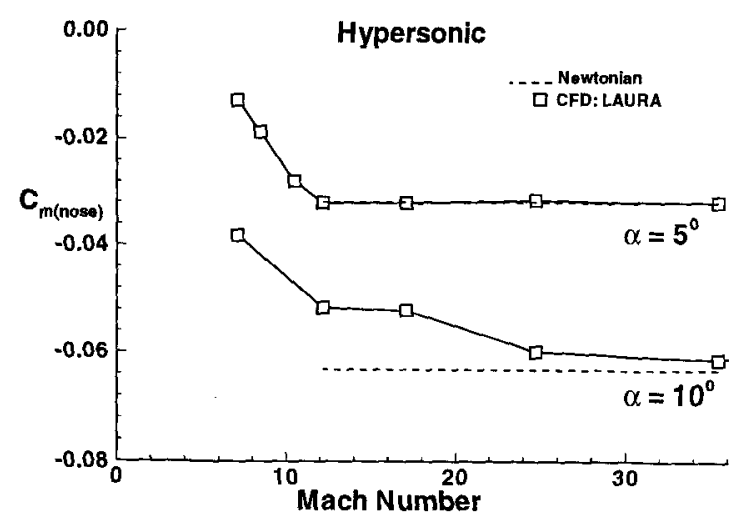

Fig. 12 Variation of the moment coefficient in the hypersonic-flow regime. 


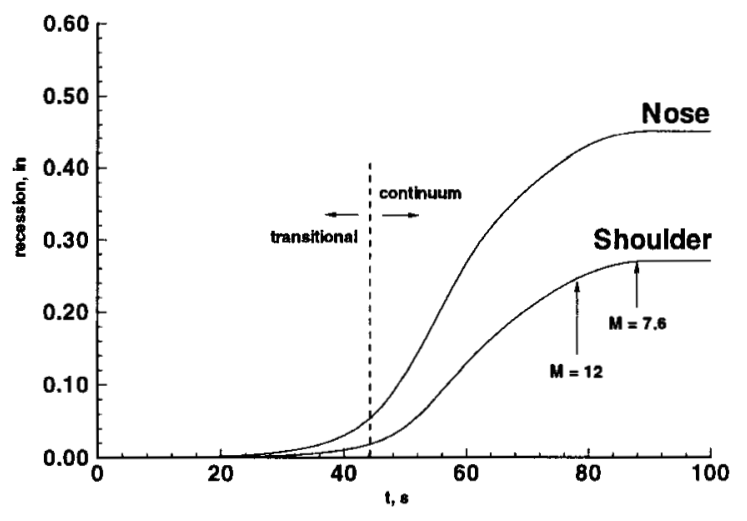

Fig. 13 Predicted surface recession during the entry trajectory.

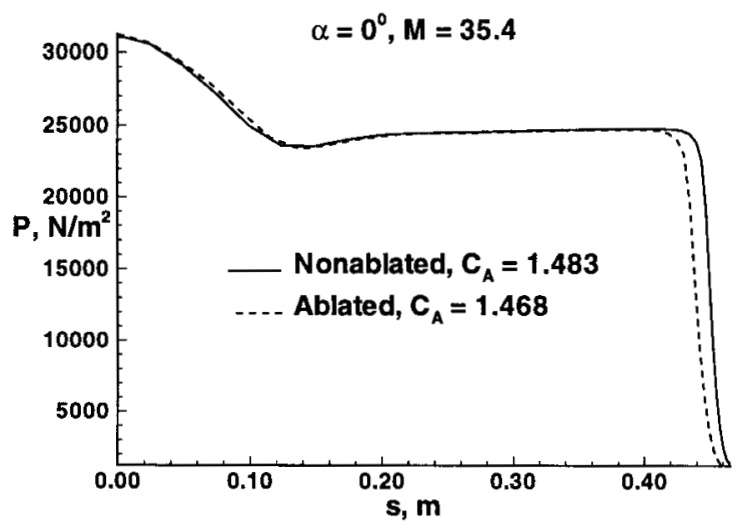

Fig. 14 Effect of surface recession on forebody pressures predictions.

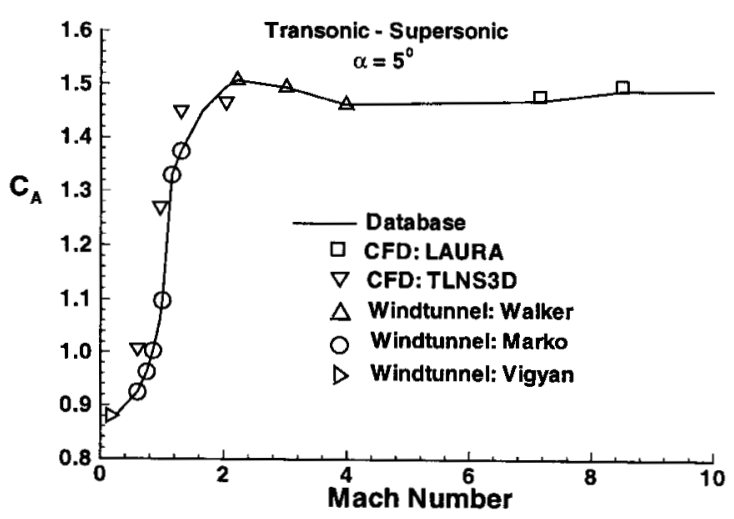

Fig. 15 Comparison of axial-force coefficient predictions in the transonic and subsonic regimes.

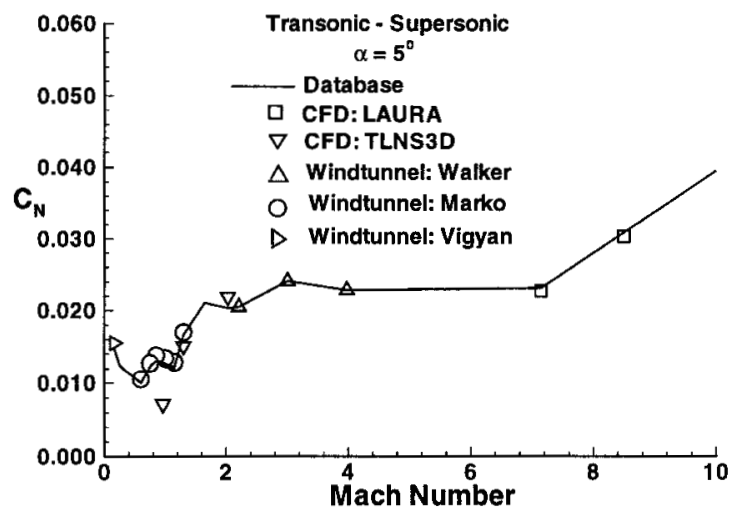

Fig. 16 Comparison of normal-force coefficient predictions in the transonic and subsonic regimes.

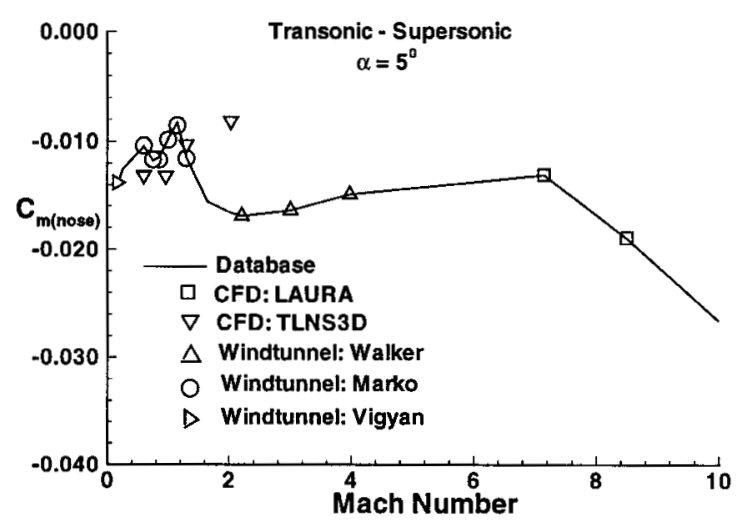

Fig. 17 Comparison of moment coefficient predictions in the transonic and subsonic regimes.

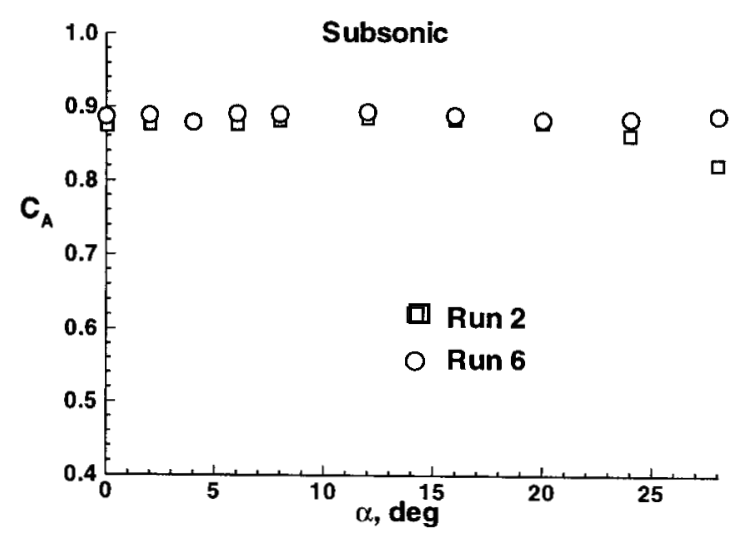

Fig. 18 Axial-force coefficient measurements at subsonic conditions $(M=0.16)$. 


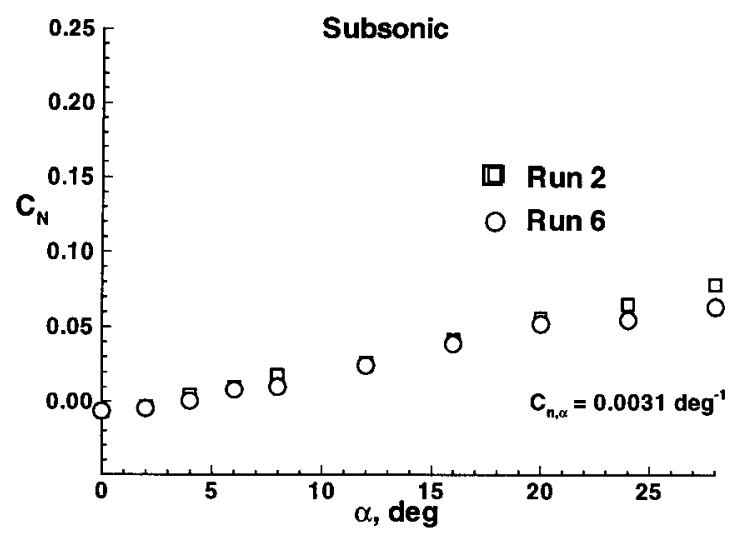

Fig. 19 Normal-force coefficient measurements at subsonic conditions $(\mathrm{M}=0.16)$.

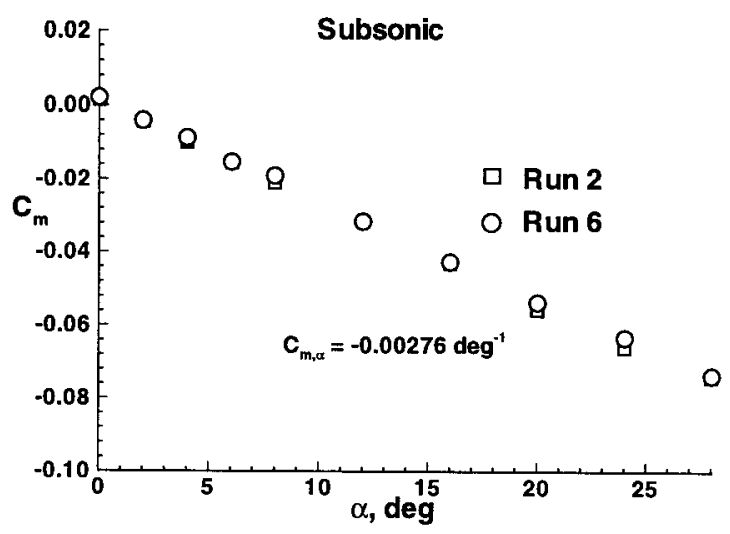

Fig. 20 Moment coefficient measurements at subsonic conditions $(M=0.16)$. 